Project 86608

\title{
Phytoremediation of Ionic and Methyl Mercury Pollution
}

\author{
Meagher, Richard B. \\ University of Georgia
}

\section{RESULTS TO DATE: PROGRESS REPORT}

\author{
ABSTRACT (INCLUDING BACKGROUND, GOALS, OBJECTIVES, AND SPECIFIC AIMS):
}

Phytoremediation is defined as the use of plants to extract, resist, detoxify, and/or sequester toxic environmental pollutants. The long-term goal of the proposed research is to develop and test highly productive, field-adapted plant species that have been engineered for the phytoremediation of mercury. A variety of different genes, which should enable plants to clean mercury polluted sites are being tested as tools for mercury phytoremediation, first in model laboratory plants and then in potential field species. Several of these genes have already been shown to enhance mercury phytoremediation. Mercury pollution is a serious, world-wide problem affecting the health of human and wildlife populations. Environmentally, the most serious mercury threat is the production of methylmercury ( $\mathrm{CH} 3 \mathrm{Hg}+)$ by native bacteria at mercury contaminated wetland sites. Methylmercury is inherently more toxic than metallic $(\mathrm{Hg}(0))$ or ionic (Hg(II)) mercury, and because methylmercury is prolifically biomagnified up the food chain, it poses the most immediate danger to animal populations. We have successfully engineered two model plants, Arabidopsis and tobacco, to use the bacterial merB gene to convert methylmercury to less toxic ionic mercury and to use the bacterial merA gene to further detoxify ionic mercury to the least toxic form of mercury, metallic mercury. Plants expressing both MerA and MerB proteins detoxify methylmercury in two steps to the metallic form. These plants germinate, grow, and set seed at normal growth rates on levels of methylmercury or ionic mercury that are lethal to normal plants. Our newest efforts involve engineering plants with several additional bacterial and plant genes that allow for higher levels of mercury resistance and mercury hyperaccumulation. The potential for these plants to hyperaccumulate mercury was further advanced by developing constitutive, aboveground, and root-specific gene expression systems. Our current strategy is to engineer plants to control the chemical speciation, electrochemical state, transport, and aboveground binding of mercury in order to manage this toxicant. To advance this mercury phytoremediation strategy, our planned research focuses on the following Specific Aims: (1) to increase the transport of mercury to aboveground tissue; (2) to identify small mercury binding peptides that enhance hyperaccumulation aboveground; (3) to test the ability of multiple genes acting together to enhance resistance and hyperaccumulation; (4) to construct a simple molecular system for creating male/female sterility, allowing engineered grass, shrub, and tree species to be released indefinitely at contaminated sites; (5) to test the ability of transgenic cottonwood and rice plants to detoxify ionic mercury and prevent methylmercury release from contaminated sediment; and (6) to initiate field testing with transgenic cottonwood and rice for the remediation of methylmercury and ionic mercury. The results of these experiments will enable the phytoremediation of methyl- and ionic mercury by a wide spectrum of deeprooted, fast-growing plants adapted to diverse environments. We have made significant progress on all six of these specific aims as summarized below.

RESEARCH PROJECT RESULTS AND IMPLICATIONS During this most recent grant period ending in summer of 2005 we focused our research on the following six specific aims, each with the direct or indirect goal of developing transgenic plants that can be released into the field and efficiently detoxify ionic and methylmercury and/or hyperaccumulate mercury. Specific Aim: (1) to increase the transport of mercury to aboveground tissue. Mercury is a chemical analog of zinc and is in the same element group in the periodic table. We propose that zinc transporters are the normal uptake system that moves mercury into plants and we wanted to enhance this process. Among nine Arabidopsis zinc transporters (ZIP) examined, we identified two that we believe exhibited strong transport of mercury. When expressed in yeast, these 
transporters produced mercury sensitive phenotypes. We believe the increased $\mathrm{Hg}(\mathrm{II})$ transport into yeast made them hypersensitive to mercury. The nine individual ZIP transporter genes tested, were generously supplied to us by Dr. Mary Lou Guerinot (Dartmouth University, NH). The two ZIP transporters that appeared to most effectively transport mercury and one control that did not appear to act on mercury were subcloned for strong constitutive plant expression and transformation into Arabidopsis. Surprisingly, the first 10 transgenic lines expressing two different ZIP transporters were significantly more resistant to 65 uM mercury in their media than wild-type. Thus, these genes confer the opposite phenotype to plants from what conferred to yeast. We believe the mechanism for this unexpected difference is based in the localization of zinc transporters to multi-membrane endosomal vesicles under conditions of zinc sufficiency. These transgenic ZIP plants are currently being compared to wild type for the ability to up take, accumulate aboveground, and volatilize mercury under conditions of zinc limitation. Transporters that are successful in moving more mercury to aboveground tissues in these assays will next be tested in the root-specific expression system described next. In the long run we need to test the expression of these transporters from a strong root-specific promoter and restrict their expression from tissues where they are not needed. Previous research on two different root-specific tobacco promoters obtained from other laboratories, as described in our original EMSP proposal, met with disappointing results. Both tobacco promoters were weak, not very root specific, and active in only a few root cell types. This resulted in our developing our own root-specific expression system. Strong, tissue-specific expression systems are essential tools in phytoremediation as in many areas of plant biotechnology. We developed a new expression system tool called a "repressor-operator gene complex" (ROC) that can be used to develop almost any tissue or organ specific pattern. ROCs have diverse applications in plant biotechnology fields including phytoremediation, disease resistance, plant nutrition, food safety, and hybrid seed production. To test this concept we assembled a root-specific ROC. When a modified E. coli lac repressor with a nuclear localization signal was expressed from a rubisco small subunit expression vector, S1pt::lacIn, LacIn protein was localized to the nuclei of leaf and stem cells, but not to root cells. A LacIn repressible Arabidopsis actin expression vector _ pot was assembled containing upstream bacterial lacO operator sequences, and it was tested using _-glucuronidase (GUS) and mercuric ion reductase (merA) gene reporters. Strong GUS enzyme expression was restricted to root tissues of A2pot::GUS/S1pt::lacIn plants, while GUS activity was high in all vegetative tissues of plants lacking the repressor. Repression of shoot activity was greater than 1000 fold for more than $25 \%$ of the plants doubly transformed with both constructs. Similarly, MerA was strongly expressed in the roots, but not the shoots of A2pot::MerA/S1pt::lacIn plants, while MerA levels remained high in both shoots and roots of plants lacking repressor. Plants with MerA expression restricted to roots were approximately as tolerant to ionic mercury as plants constitutively expressing MerA. The data on the root-specific ROC and the ROC concept is in press in the Plant Biotechnology journal (Kim et al., 2005). Our root-specific ROC significantly outperformed both of the root-specific tobacco promoters we had tried previously and the data for one of these is shown for comparison in this manuscript. Specific Aim: (2) to identify small mercury binding peptides that enhance hyperaccumulation aboveground. We characterized three genes encoding the necessary enzymes in the phytochelatin synthesis pathway (ECS, GS, PCS). Expression of any one of these enzymes confers low levels of mercury resistance to plants, and combinations of these enzymes provide even higher moderate levels of resistance. We are preparing several manuscripts for publication describing these results and two have been published in the last grant period ( $\mathrm{Li}$ and Redei, 1969; Li et al., 2004; Li et al., 2005; Li et al., in prep.-b). In addition, we have begun to characterize the expression of three new genes that will make mercury binding macromolecules, MerP and polyphosphate kinase (PPK). Specific Aim: (3) to test the ability of multiple genes acting together to enhance resistance and hyperaccumulation. Over the last decade we have developed a constitutive plant actin 2 promoter system (A2pt) in our laboratory that expresses very high levels of target proteins (An et al., 1996; Dhankher et al., 2002). Our data suggests that the promoter expresses about 5 times more of most target proteins than the enhanced 35S CaMV viral promoter. This promoter and the 35S promoter have been used to express genes encoding more than 25 different target enzymes with potential applications to mercury remediation. Earlier EMSP funded research demonstrated significant 100 to 1000 fold gains in methylmercury processing rates when MerB and MerA were combined (Bizily et al., 2000; Bizily et al., 2003). During the last grant period we tested the following gene combinations in Arabidopsis and each produced moderate or significant gains in mercury resistance or sequestration (MerB/ECS; ECS/GS; ECS/PCS; GS/PCS; ECS/GS/PCS). While these single gene combinations ECS, GS, or PCS conferred high levels of arsenic resistance, none conferred either high levels of mercury resistance or resulted in high levels of mercury accumulation in target tissues. In contrast, the co-expression of ECS and GS conferred 3- 
fold increase in Hg accumulation above ground. Extensive data have been collected on ECS x GS plants and manuscript is being prepared for publication (Li et al., in prep.-a). A few of the two and three combinations are being examined in more detail and will be moved into cottonwood for field-testing. The competitive renewal we submitted a few months ago describes in detail our plans to make plants coexpressing several transgenes. Specific Aim: (4) to construct a simple molecular system for creating male/female sterility, allowing engineered grass, shrub, and tree species to be released indefinitely at contaminated sites. We have outstanding data on a novel male/female sterility system to allow the longterm release of transgenic plants into the environment for phytoremediation. Completely sterile transgenic plants would require less field management and lower cost because these sterile plants would not be able to pass transgenes into wild plant populations nor could they receive pollen from native plants and make seed. We have shown that the actin 11 and actin 12 promoters are expressed strongly, but almost exclusively, in both male and female and in male reproductive tissues, respectively (Huang et al., 1996; Huang et al., 1997). Based on these data and as outlined in our DOE EMSP proposal, we developed A11pt and A12pt expression vectors and used RNA interference (RNAi) and knocked down the expression of multiple reproductive actin genes. However, while all of these plants were partially sterile, none were completely sterile. As an alternative we used these vectors and a RNAi approach to develop thiamine deficiency based sterility (TDS). We silenced the Arabidopsis Thi2 and Thi3 genes, which encode essential enzymes in thiamin biosynthesis. Nearly all of the 60 T1 generation A11pt::Thi2 and A11pt::Thi3 transgenic plants were partially or fully sterile (6 completely sterile plants). One hundred percent of the T1 generation A12pt::Thi3 plants (7 of 7 plants) were fully male-sterile (produced no seed). These results are being quantified and will be prepared for publication (Kim and Meagher, in preparation). Collaborative projects have been initiated to test TDS in other plant species. Specific Aim: (5) to test the ability of transgenic cottonwood, tobacco, and rice plants to detoxify ionic mercury and prevent methylmercury release from contaminated sediment. Our basic research projects on transgenic cottonwood, tobacco, and rice expressing the merA or merB gene as described in our proposal have been completed and recently published or submitted for publication (Che et al., 2003; Heaton et al., 2003; Heaton et al., in press; Che et al., submitted). Our research on cottonwood is performed collaboratively with Professor Scott Merkle in the School of Forest Resources at UGA. Recent data on cottonwood expressing merA and merB are excellent and these plants are being propagated as they are being characterized for mercury processing (Lyyra et al., in prep.). It will take several more months to have enough plant material to begin soil tests in the greenhouse. With Dr. Merkle we also have developed transgenic MerA/MerB sweetgum. Sweetgum thrives in some extremely wet habitats, where methylmercury is a problem and where cottonwood might not grow well. We are in the process of propagating the transgenic shoots of these plants for further analysis. Specific Aim: (6) to initiate field testing with transgenic cottonwood and rice for the remediation of methylmercury and ionic mercury. Transgenic merA expressing cottonwood have been planted at two mercury-contaminated field sites and we will describe one site in detail. At the site of an abandoned hat factory that has been contaminated with mercury for more than a hundred years we planted 120 merA cottonwood and 60 wild type cottonwood. The site was divided into four large plots ranging from 30 to $1000 \mathrm{ppm}$ of mercury in the soil. Each plot was divided into 12 barrel sized subplots, that received 3 plants each of the same genotype. Initial assays of mercury content have been made from these plots at the time of summer planting. The plants are now 8 to $10 \mathrm{ft}$ tall. Plant (stem and leaf) and soil mercury content is being assayed. While these are the least sophisticated of the transgenic plants we have planned to use in the field, we still expect some reduction in soil mercury content. The long delay from model plants in the to field work results from the inherent slow transformation and regeneration of trees and the fact that it takes several months to a year to go through the APHIS system and obtain a Permit for field testing of transgenic trees in the field. 